\title{
TOMOGRAFIA COMPUTADORIZADA HELICOIDAL DOS SEIOS PARANASAIS NA CRIANÇA: AVALIAÇÃO DAS SINUSOPATIAS INFLAMATÓRIAS*
}

\author{
Luiz Dias Dutra ${ }^{1}$, Edson Marchiori ${ }^{2}$
}

\begin{abstract}
Resumo Neste estudo foi feita avaliação retrospectiva de 71 casos selecionados de pacientes pediátricos, maiores de um ano e menores de sete anos, que realizaram exame de tomografia computadorizada helicoidal dos seios paranasais no período de março de 1997 a abril de 1998, apresentando quadro clínico de sinusopatia inflamatória aguda recorrente e sinusopatia inflamatória crônica. Foram correlacionados os quadros clínicos com os achados da tomografia computadorizada helicoidal, avaliando a pneumatização dos seios paranasais, os complexos óstio-meatais, as variações anatômicas, o estado da superfície mucosa e a extensão lesional. Os achados tomográficos de maior prevalência foram o velamento total ou parcial de uma ou mais cavidades paranasais $(92,9 \%)$, seguido da hipertrofia da mucosa de revestimento $(67,6 \%)$. Houve, na maioria dos casos, associação entre a sinusopatia inflamatória e a obstrução dos complexos óstio-meatais (53,5\%). As variações anatômicas foram identificadas já a partir de um ano, com predomínio dos desvios do septo nasal $(14,1 \%)$, estando correlacionadas às sinusopatias inflamatórias em cerca de $71 \%$ dos pacientes. Unitermos: Tomografia computadorizada helicoidal. Seios paranasais. Radiologia.
\end{abstract}

\begin{abstract}
Helical computed tomography of the paranasal sinuses in children: evaluation of sinus inflammatory diseases.

We conducted a retrospective analysis of 71 selected pediatric patients, aged 1 to 7 years, which were submitted to helical computed tomography examination of the paranasal sinuses in the period between March, 1997 and April, 1998 due to recurrent acute sinusitis and chronic sinusitis. Clinical and helical computed tomography findings were compared by evaluating the pneumatization of the paranasal sinuses, ostiomeatal complexes, anatomic variants, the status of mucosal surface and lesion extension. The most common CT findings were total or partial opacification of one or more paranasal cavities $(\mathbf{9 2 . 9 \%})$, followed by mucosal thickening $(67.6 \%)$. In the majority of cases there was association between sinusitis and ostiomeatal obstruction. Anatomic variants were identified from the age of one year. The most common anatomic variant was septonasal deviation $(14.1 \%)$ that was associated to sinusitis in about $71 \%$ of the patients.

Key words: Helical computed tomography. Paranasal sinuses. Radiology.
\end{abstract}

\section{INTRODUÇÃO}

A tomografia computadorizada (TC) é, atualmente, a modalidade de escolha entre os métodos de imagem para a avaliação dos seios paranasais e das estruturas adjacentes ${ }^{(1)}$. Sua capacidade em demonstrar e diferenciar as estruturas ósseas, os tecidos moles e o ar permite uma avaliação minu-

* Trabalho realizado no Hospital Infantil Luís França e no Serviço de Radiodiagnóstico do Hospital Universitário Clementino Fraga Filho (HUCFF) da Universidade Federal do Rio de Janeiro (UFRJ), Rio de Janeiro, RJ.

1. Mestrando em Radiologia da UFRJ, Ex-Estagiário do Service de Radiologie - Hôpital d'Enfant Armand Trusseau, Paris, France.

2. Professor Titular de Radiologia da Universidade Federal Fluminense (UFF), Coordenador Adjunto do Curso de Pósgraduação em Radiologia da UFRJ.

Endereço para correspondência: Prof. Dr. Edson Marchiori. Rua Thomaz Cameron, 438, Valparaíso. Petrópolis, RJ 25685-120. E-mail: edmarchiori@zipmail.com.br

Recebido para publicação em 26/11/2001. Aceito, após revisão, em 3/12/2001. ciosa da anatomia, das variações anatômicas e da presença e extensão de lesões intra e extra-sinusais ${ }^{(2)}$.

A TC helicoidal (ou espiral ou de aquisição volumétrica) é a última inovação tecnológica neste segmento de aparelhos de imagem, tendo sido introduzida na prática clínica em $1989^{(3)}$. Esta técnica baseia-se na aquisição de volume de uma determinada região e permite a realização de reconstruções multiplanares e tridimensionais de ótima qualidade. A rapidez dessas aquisições volumétricas faz com que os exames sejam realizados em tempos curtos (geralmente abaixo de 30 segundos), o que, por si só, demonstra a sua utilidade no campo pediátrico.

A sinusopatia inflamatória é, atualmente, considerada a doença crônica de maior prevalência em todas as faixas etárias e a quinta maior causa de uso de antibióticos ${ }^{(4)}$.
Na faixa etária avaliada neste trabalho (crianças maiores de um ano e menores de sete anos) é freqüente a ocorrência das sinusopatias inflamatórias agudas recorrentes (SIAR) e sinusopatias inflamatórias crônicas (SIC), e o tratamento habitual é o uso de antibioticoterapia, muitas vezes prolongada, com os riscos inerentes à sua utilização (nefrotoxicidade, ototoxicidade, etc. $)^{(5)}$. Estas crianças apresentam, muitas vezes, dificuldades no convívio social e no seu desenvolvimento, pelo quadro clínico apresentado.

A cirurgia endoscópica endonasal representa a mais avançada forma de tratamento das SIAR e SIC, mesmo no grupo pediátrico, e o papel da TC é fundamental no planejamento cirúrgico, servindo como guia anatômico para a intervenção ${ }^{(6)}$.

Neste trabalho, a técnica de exame apresentada é de grande valor, não somente por 
demonstrar a existência de lesões mucosas nos seios paranasais e a sua extensão, mas também por demonstrar com clareza toda a anatomia dos seios paranasais e das cavidades nasais, por meio das reconstruções multiplanares, e, assim, permitir o planejamento cirúrgico seguro para as crianças selecionadas para este tipo de tratamento.

\section{CASUÍSTICA E MÉTODOS}

No período compreendido entre março de 1997 e abril de 1998, foram avaliadas 80 crianças com idade variando de um a seis anos, que realizaram TC helicoidal para a avaliação dos seios paranasais e outras regiões anatômicas correlacionadas, em um hospital infantil (Hospital Infantil Luís França), na cidade de Fortaleza, CE. Foram excluídas as crianças que apresentavam anomalia crânio-facial ou quadro de imunossupressão de qualquer natureza, restando uma população de 71 crianças.

Os exames foram realizados em equipamento Toshiba, modelo X-Vision, aparelho do tipo espiral, capaz de realizar aquisições volumétricas com posteriores reconstruções multiplanares. As aquisições foram realizadas sempre no plano axial, com os pequenos pacientes em decúbito dorsal, utilizando-se como parâmetros técnicos: 50 $\mathrm{mAs} / 120 \mathrm{kV}$ (valores mínimos aceitos pelo referido aparelho), filtro de reconstrução para osso, espessura de corte de $2 \mathrm{~mm}$, "pitch" de 1; o campo de visão (FOV) era o menor possível e o "gantry" era sempre mantido sem qualquer inclinação. Após as aquisições das imagens, inicialmente eram feitas reconstruções destas imagens primitivas axiais com intervalo entre os cortes de $1 \mathrm{~mm}$ e com ampliação suficiente para a avaliação não somente dos seios paranasais bem como de outras estruturas correlatas (orelhas, vegetações adenóides, amígdalas palatinas, etc.).

Posteriormente, realizavam-se as reconstruções multiplanares nos planos coronal (avaliando sempre os complexos óstio-meatais), sagital (com atenção à rinofaringe e à orofaringe), e eventualmente em planos oblíquos.

Tratando-se de crianças de baixa idade, a sedação era eventualmente necessária, embora o tempo de exame fosse exíguo (média de 20 segundos de aquisição e cer- ca de três minutos para toda a preparação e execução do exame). A sedação ocorreu sobretudo no grupo etário compreendido entre um e quatro anos. As crianças acima de quatro anos raramente foram sedadas, sendo em geral suficientes a presença de um dos pais na sala de exame e o preparo psicológico da criança. A sedação foi realizada com utilização de hidrato de cloral a $10 \%$, na dosagem de $50 \mathrm{mg} / \mathrm{kg} /$ dose, por via retal, sendo a dose máxima (mesmo nos casos de doses repetidas) de $2.000 \mathrm{mg}$. Os pacientes eram monitorizados com oxímetro de pulso, com controle permanente da freqüência cardíaca e das saturações gasosas sanguíneas. Durante este trabalho não ocorreu qualquer reação indesejada ou qualquer efeito colateral nestas crianças, independentemente da idade. Episódios de evacuação pós-introdução da sonda retal foram esporádicos, sem maiores conseqüências. Os pais eram sempre alertados sobre o medicamento administrado e também como proceder após o exame, sobretudo em relação à alimentação.

Em nenhum dos exames realizados foi feita administração venosa de meio de contraste iodado.

Os exames foram solicitados por médicos assistentes (na sua grande maioria pediatras), em crianças com sintomatologia compatível com SIAR ou SIC. Na maioria das vezes estas crianças já se encontravam em tratamento clínico quando da realização do exame tomográfico, com média de 14 dias de antibioticoterapia e uso de antiinflamatórios.

O protocolo seguido incluía dados dos pacientes sobre idade, sexo, quadro clínico e duração da sintomatologia, totalizando 71 casos que constituem a casuística deste trabalho.

A divisão dos pacientes foi feita essencialmente com base na faixa etária (maiores de um ano e menores de sete anos), agrupando-se as crianças segundo os sintomas apresentados, ou seja, com sinusite aguda recorrente ou crônica.

Foram considerados como seios desenvolvidos aqueles que apresentavam qualquer grau de aeração.

Os termos velamento ou opacificação sinusal foram empregados para se referir ao preenchimento da luz sinusal por material hipoatenuante, com densidade média se- melhante àquela dos tecidos moles. A hipertrofia da mucosa foi classificada em leve, média ou acentuada, segundo o grau de espessamento desta mucosa em relação ao volume sinusal ${ }^{(7)}$. Assim, consideramos como leve se a hipertrofia envolvia até 33\% do volume do seio analisado, moderada até $67 \%$ e acentuada quando acima de $67 \%$. Os complexos óstio-meatais foram analisados, bem como as principais variações anatômicas, sendo que estes dois últimos dados são atualmente considerados de grande importância na gênese das sinusopatias inflamatórias recorrentes e crônicas, sendo passíveis de intervenções cirúrgicas.

\section{RESULTADOS}

\section{Achados clínicos e epidemiológicos}

A população do estudo, com faixa etária compreendendo crianças maiores de um ano e menores que sete anos, foi distribuída em intervalos de um ano de idade.

Na população examinada houve predomínio de casos entre as crianças maiores de três anos e menores de quatro anos (22 pacientes; 31\%), seguidas do grupo composto por pacientes maiores de dois anos e menores de três anos (19 pacientes; 26\%).

Em relação ao sexo, houve predomínio do masculino (46 pacientes; 64,7\%) em relação ao feminino (25 pacientes; 35,3\%).

Os pacientes analisados neste estudo apresentaram como sintomas mais comuns secreção e obstrução nasais $(76 \%)$, tosse crônica $(25,3 \%)$ e febre $(25,3 \%)$, podendo ser concomitantes ou não. A secreção nasal era quase sempre purulenta e freqüentemente associada com edema da mucosa nasal e congestão. Estes achados foram mais prevalentes nas crianças menores de quatro anos.

Outros achados clínicos foram observados, porém o número de ocorrências era insignificante. Algumas vezes estes achados ou sintomas estavam correlacionados com a sinusopatia, outras vezes representavam doenças de base e a sinusopatia não era o quadro principal. Dentre eles, podem ser citados vômitos, discinesia ciliar e polipose nasossinusal.

Muitas crianças com sinusite crônica têm história importante de associação com otite média recorrente ou otite média com efusão. 
Neste estudo observamos um índice de $18,3 \%$ no total de crianças acometidas por SIAR e SIC que apresentavam efusão nas orelhas médias (otite média secretora) ou outras alterações relacionadas às otites agudas ou agudas recorrentes, com distribuição porcentual semelhante nas diversas faixas de idade.

A hipertrofia das vegetações adenóides foi outro achado relevante neste trabalho, freqüente em todas as faixas etárias analisadas (48 casos; 67,8\%). As dimensões do tecido adenoidiano foram medidas no plano sagital, fazendo-se correspondência entre a altura do corpo esfenoidal e a maior espessura do tecido adenoidiano observada neste plano. Considerou-se como dentro dos limites da normalidade quando a maior medida da espessura do tecido adenoidiano foi no máximo igual à maior altura do corpo do esfenóide, sem determinar obstrução da região coanal posterior.

\section{Achados radiológicos}

Dos exames das 71 crianças selecionadas, $16(22,5 \%)$ foram considerados normais, ou seja, não apresentavam qualquer alteração da mucosa de revestimento dos seios paranasais ou na região dos complexos óstio-meatais. Embora em algumas crianças não houvesse lesão mucosa nos seios paranasais, elas apresentavam outras alterações como hipertrofia das vegetações adenóides (oito casos; $11,2 \%$ ) ou hipertrofia das amígdalas palatinas (três casos;
$4,2 \%$ ), que algumas vezes poderiam simular quadro de sinusopatia inflamatória.

A aeração dos seios paranasais apresentou-se em diferentes fases de desenvolvimento, segundo a idade. Os seios maxilares e etmoidais estavam pneumatizados nas 71 crianças avaliadas (100\% dos casos).

Os seios do esfenóide, cuja pneumatização inicia-se em torno dos seis meses, apresentaram aeração com porcentuais crescentes em relação às faixas etárias, tendo sido observada em apenas um dos sete pacientes com idade entre um e dois anos $(14,3 \%)$ e em todas as crianças (100\%) com idade entre seis e sete anos.

Embora a aeração dos seios do frontal comece em geral em torno dos dois a três anos, ela só foi observada a partir dos quatro anos.

No que se refere às variações anatômicas, muitas vezes relacionadas aos quadros crônicos ou recorrentes de sinusite, por determinarem disfunções na ventilação e drenagem de secreções dos complexos óstio-meatais, os desvios do septo nasal apresentaram a maior prevalência (dez casos; $14,1 \%$ ), seguidos de concha média bullosa (três casos; 4,2\%), aeração da crista galli (dois casos; 2,8\%), células de Haller (um caso; $1,4 \%$ ) e variações da apófise unciforme (um caso; 1,4\%) (Figuras 1, 2, 3 e 4).

Os achados tomográficos mais prevalentes relacionados às sinusopatias inflamatórias foram o velamento total ou parcial de um ou mais seios paranasais (66 casos; 92,9\%) e a hipertrofia da mucosa de revestimento sinusal (48 casos; 67,6\%). Em muitos destes pacientes os achados tomográficos foram concomitantes (dez casos; $18,8 \%$ ) e envolviam mais de um seio paranasal.

Em relação ao velamento total ou parcial de seio(s) paranasal(is), que foi o achado tomográfico mais freqüente, observamos que os seios maxilares $(n=18 ; 25,3 \%)$ e etmoidais $(n=46 ; 64,8 \%)$ foram os mais envolvidos, este último com maior prevalência.

Os seios esfenoidais foram menos acometidos ( $\mathrm{n}=2 ; 2,8 \%$ ). Não constatamos acometimento dos seios do frontal (Figuras 5, 6, 7 e 8).

Os velamentos sinusais foram mais comuns nas crianças na faixa etária de três a quatro anos (Tabela 1).

O outro achado radiológico relevante, a hipertrofia da mucosa de revestimento dos seios paranasais, foi observado em 48 pacientes $(67,6 \%)$. Na maioria das vezes esta hipertrofia mucosa apresentava relevo irregular e era de grau moderado, ou seja, envolvia no máximo $67 \%$ do volume do respectivo seio paranasal. Houve maior prevalência de acometimento dos seios maxilares (Tabela 2) (Figuras 9 e 10).

As obstruções dos complexos óstiomeatais, associadas ou não às variações anatômicas adjacentes, deveram-se a lesões da mucosa de revestimento, sendo estas locais (p. ex.: hipertrofia mucosa focal envol-

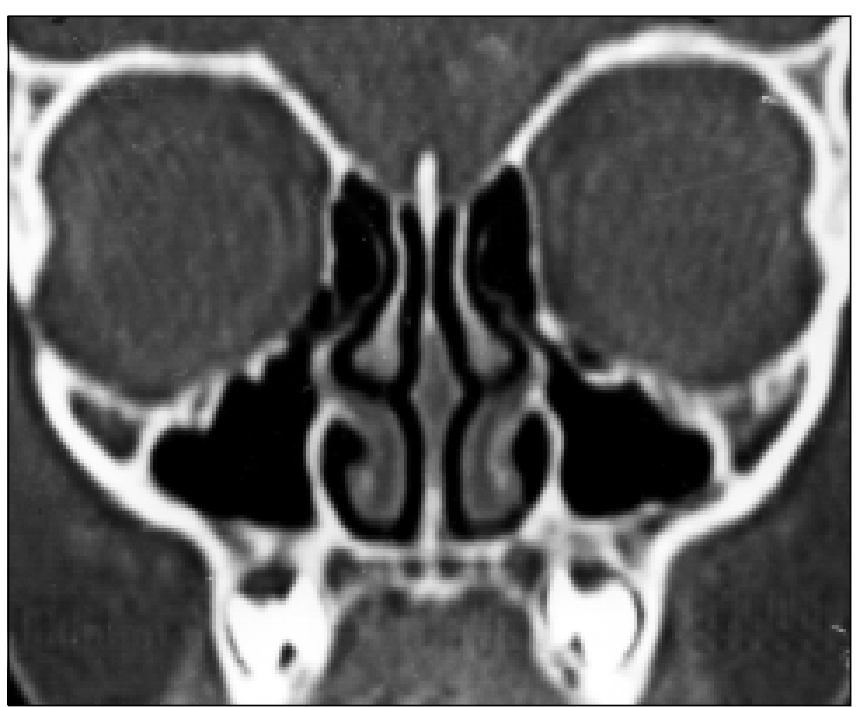

Figura 1. Reconstrução no plano coronal evidenciando células de Haller à esquerda, reduzindo as dimensões dos desfiladeiros óstio-infundibulares.

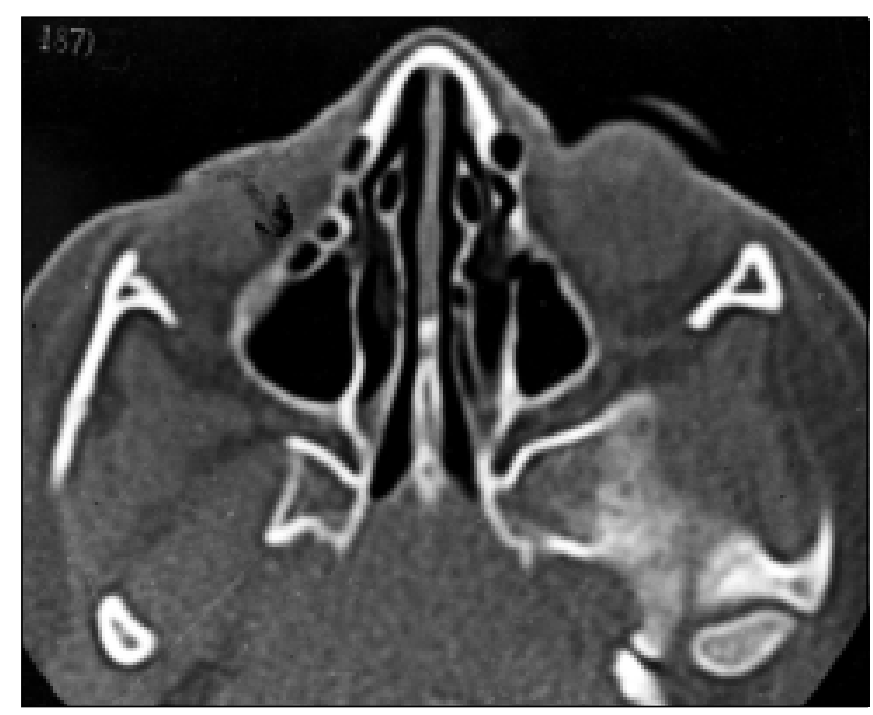

Figura 2. Imagem no plano axial demonstrando células de Haller à direita. Observa-se também aeração dos cornetos médios (variantes anatômicas). 


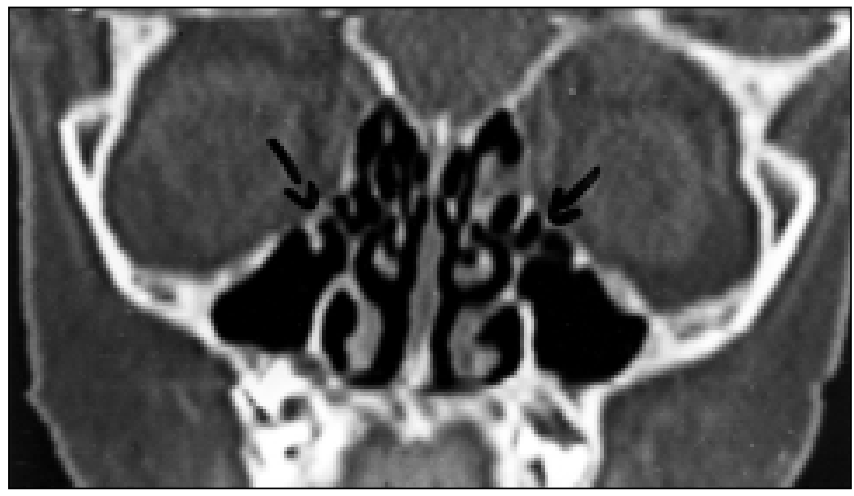

Figura 3. Reconstrução no plano coronal evidenciando células de Haller bilaterais, reduzindo as dimensões dos desfiladeiros óstio-infundibulares, e cornetos médios de morfologia "paradoxal". São variantes anatômicas que podem dificultar a drenagem mucociliar normal nos complexos óstio-meatais.

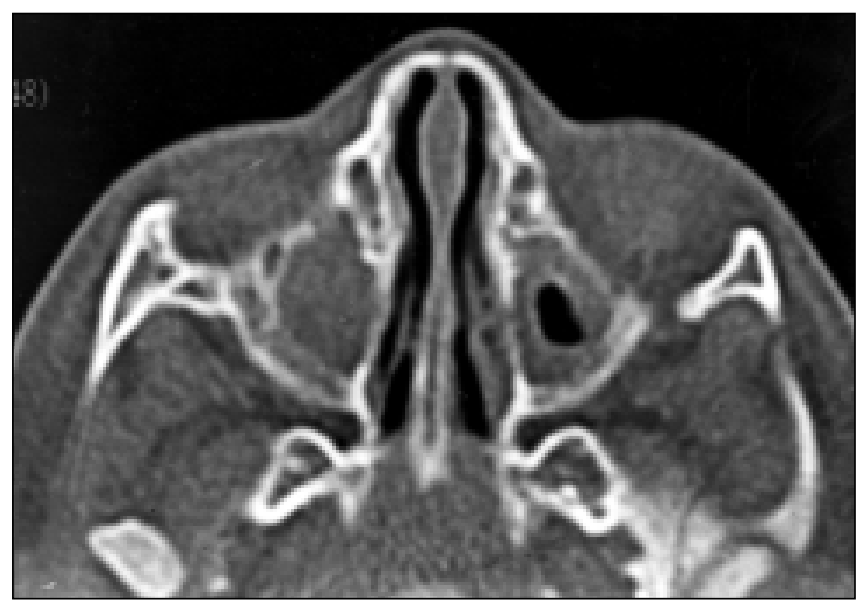

A

Figura 5. Em A plano axial, existe velamento total do seio maxilar direito e hipertrofia circunscrita da mucosa de revestimento do seio maxilar esquerdo. Em B, plano coronal, observa-se velamento das células etmoidais bilaterais. Sinusopatia inflamatória maxilo-etmoidal.

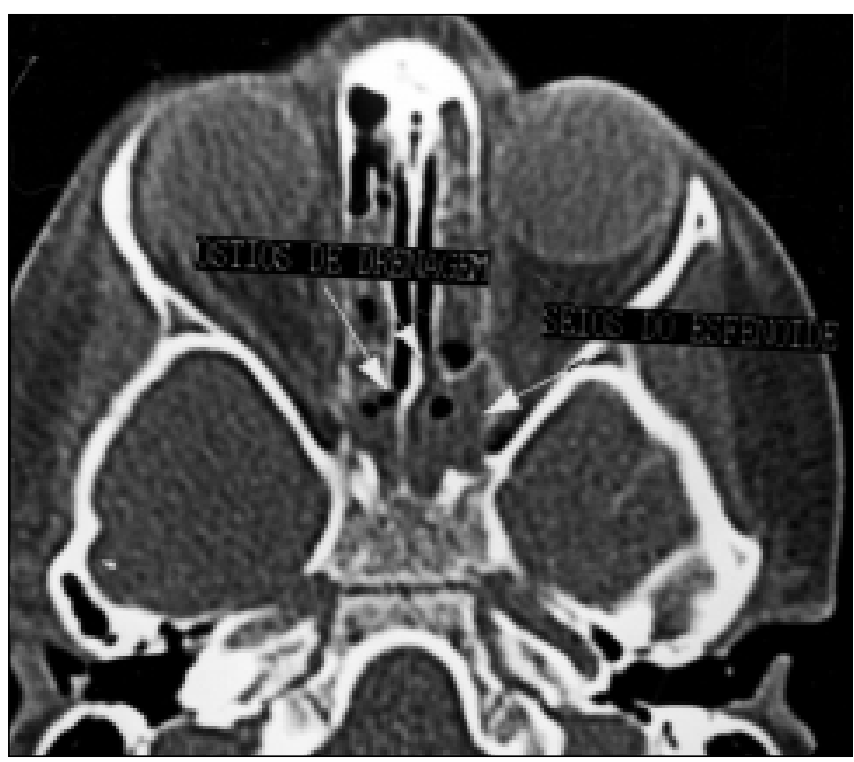

Figura 6. Plano axial. Seios esfenoidais parcialmente aerados e com velamento quase total da sua luz, determinado por importante hipertrofia da mucosa de revestimento. Sinusopatia inflamatória.

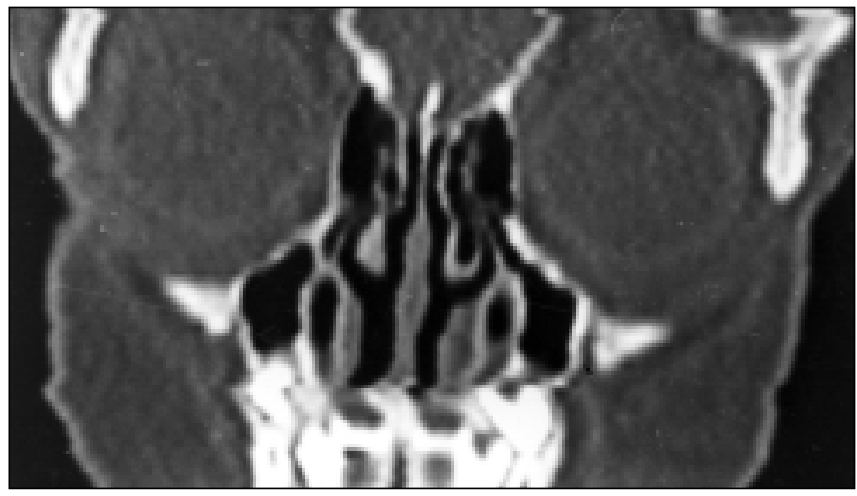

Figura 4. Reconstrução no plano coronal. Corneto médio totalmente aerado à esquerda ("concha média bullosa"), reduzindo a luz do meato médio ipsilateral.

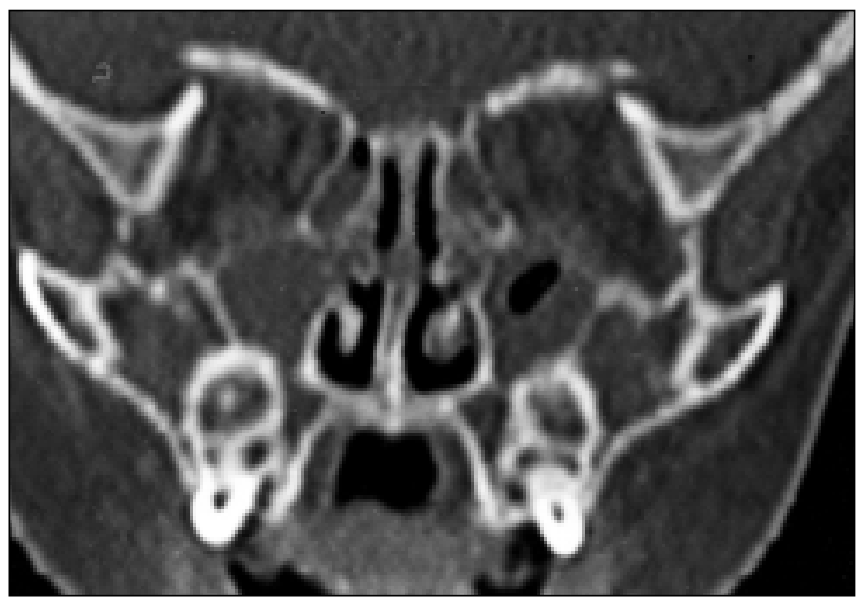

B

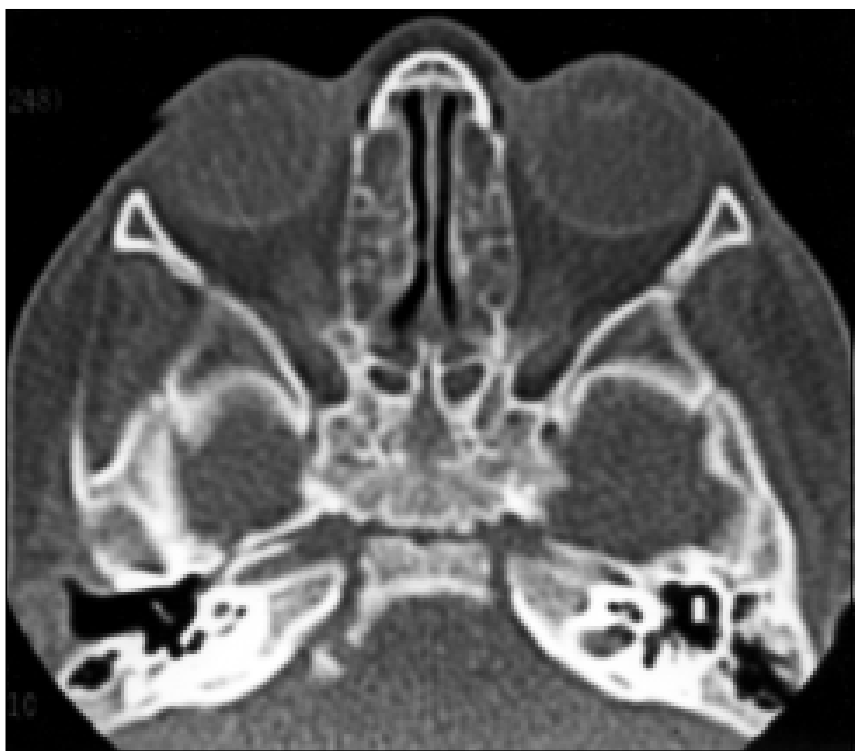

Figura 7. Plano axial. Velamento das células do labirinto etmoidal e dos seios do esfenóide. Sinusopatia inflamatória esfeno-etmoidal. 


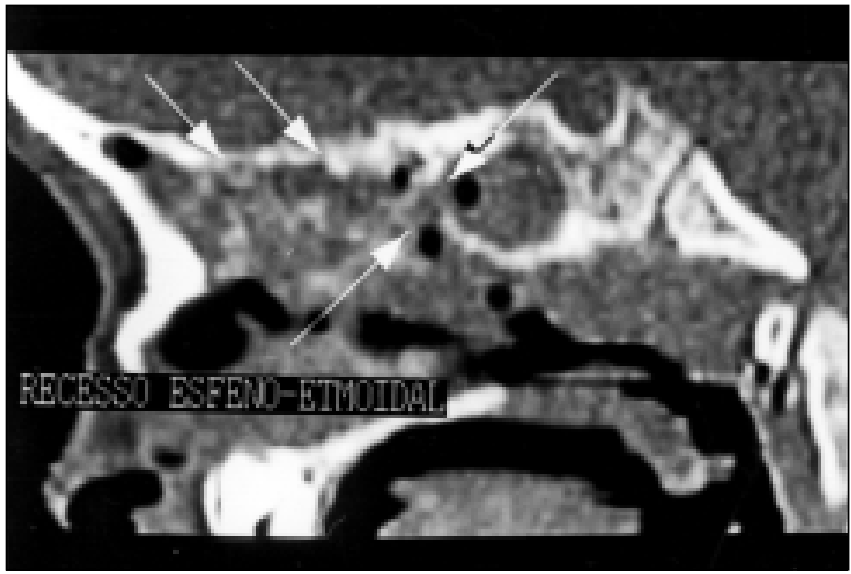

8

Figura 8. Reconstrução no plano sagital. Opacificação difusa das células etmoidais e do seio esfenoidal. Recesso esfeno-etmoidal individualizado, que é a via de drenagem das células etmoidais posteriores e do seio esfenoidal.

Figura 9. Plano coronal. Hipertrofia de grau moderado da mucosa dos seios maxilares e velamento do labirinto etmoidal, asssociados a obstrução dos complexos óstio-meatais, por espessamento mucoso loco-regional (sinusite de padrão obstrutivo dos complexos óstio-meatais). Importante desvio do septo nasal para a esquerda (variante anatômica).

Figura 10. Plano coronal. Hipertrofia de leve intensidade e com superfície irregular da mucosa de revestimento do seio maxilar direito (sinusopatia inflamatória maxilar). Observa-se também sinuosidade do septo nasal (variante anatômica).

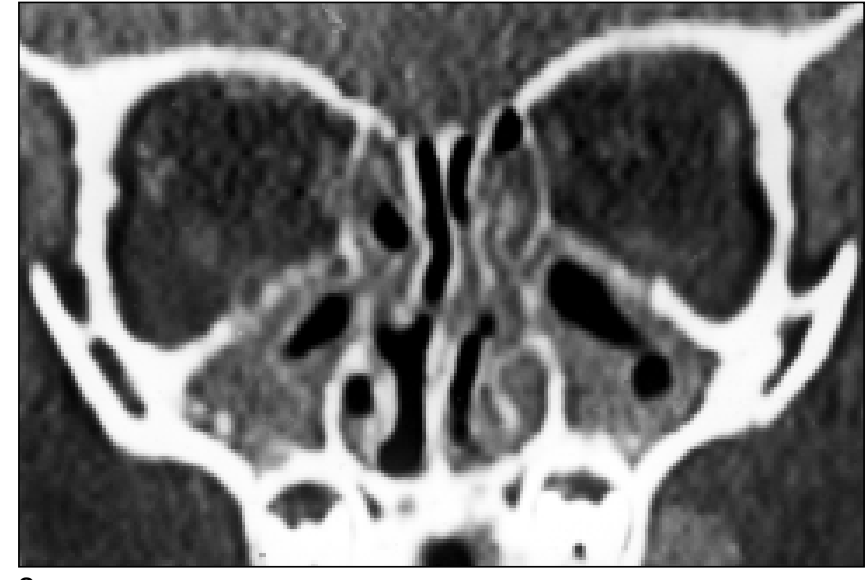

9

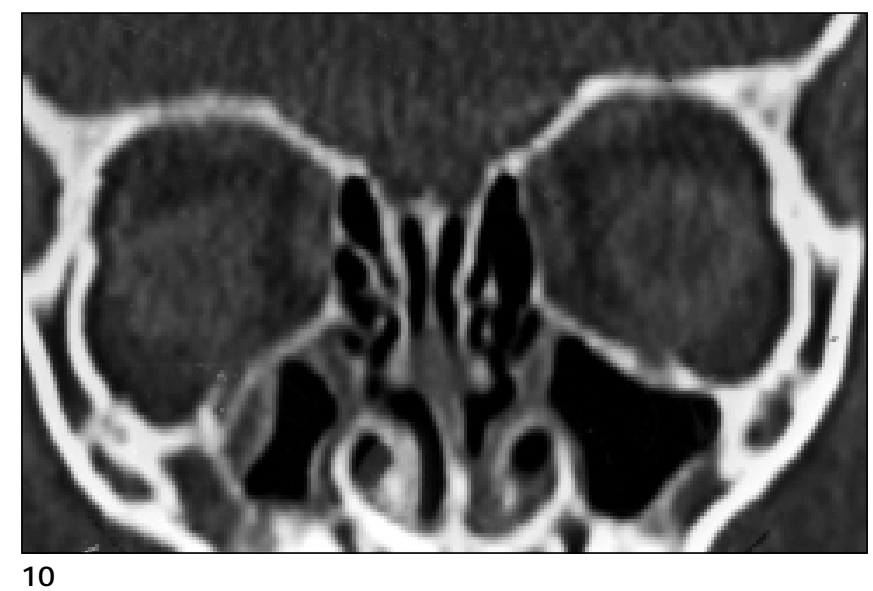

Tabela 1 Prevalência de velamento total ou parcial do(s) seio(s) paranasal(is) por faixa etária.

\begin{tabular}{|l|rc|rc|rc|cc|cr|}
\hline \multirow{2}{*}{$\begin{array}{c}\text { Faixa } \\
\text { etária }\end{array}$} & \multicolumn{2}{|c|}{ Maxilares } & \multicolumn{2}{|c|}{ Etmoidais } & \multicolumn{2}{|c|}{ Esfenoidais } & \multicolumn{2}{|c|}{ Frontais } & \multicolumn{2}{|c|}{ Total } \\
\cline { 2 - 13 } & $\mathrm{N}$ & $\%$ & $\mathrm{~N}$ & $\%$ & $\mathrm{~N}$ & $\%$ & $\mathrm{~N}$ & $\%$ & $\mathrm{~N}$ & $\%$ \\
\hline $1<2$ & 4 & 57,1 & 5 & 71,4 & - & - & - & - & 7 & 9,8 \\
$2<3$ & 3 & 15,8 & 12 & 63,1 & 1 & 5,2 & - & - & 19 & 26,7 \\
$3<4$ & 5 & 22,7 & 15 & 68,2 & - & - & - & - & 22 & 30,9 \\
$4<5$ & 2 & 18,2 & 6 & 60,0 & 1 & 10,0 & - & - & 10 & 14,0 \\
$5<6$ & 3 & 27,2 & 7 & 63,6 & - & - & - & - & 11 & 15,5 \\
$6<7$ & 1 & 50,0 & 1 & 50,0 & - & - & - & - & 2 & 2,5 \\
\hline Total & 18 & 25,3 & 46 & 64,8 & 2 & 2,8 & - & - & 71 & 100,0 \\
\hline
\end{tabular}

Obs.: Em alguns casos havia acometimento concomitante de mais de um grupo de seios paranasais.

Tabela 2 Prevalência de hipertrofia mucosa dos seios paranasais por faixa etária.

\begin{tabular}{|l|rr|rr|rr|rr|rr|}
\hline \multirow{2}{*}{$\begin{array}{c}\text { Faixa } \\
\text { etária }\end{array}$} & \multicolumn{2}{|c|}{ Maxilares } & \multicolumn{2}{|c|}{ Etmoidais } & \multicolumn{2}{|c|}{ Esfenoidais } & \multicolumn{2}{|c|}{ Frontais } & \multicolumn{2}{|c|}{ Total } \\
\cline { 2 - 14 } & $\mathrm{N}$ & $\%$ & $\mathrm{~N}$ & $\%$ & $\mathrm{~N}$ & $\%$ & $\mathrm{~N}$ & $\%$ & $\mathrm{~N}$ & $\%$ \\
\hline $1<2$ & 3 & 42,8 & 1 & 14,2 & - & - & - & - & 7 & 9,8 \\
$2<3$ & 9 & 47,3 & - & - & 1 & 5,3 & - & - & 19 & 26,7 \\
$3<4$ & 12 & 54,5 & - & - & 7 & 31,8 & - & - & 22 & 30,9 \\
$4<5$ & 4 & 40,0 & - & - & 3 & 30,0 & 1 & 10,0 & 10 & 14,0 \\
$5<6$ & 3 & 27,3 & - & - & 5 & 45,4 & - & - & 11 & 15,5 \\
$6<7$ & - & - & - & - & - & - & - & - & 2 & 2,5 \\
\hline Total & 31 & 43,6 & 1 & 1,4 & 16 & 22,5 & 1 & 1,4 & 71 & 100,0 \\
\hline
\end{tabular}

Obs.: Em alguns casos havia acometimento concomitante de mais de um grupo de seios paranasais. vendo o meato médio, com velamento de seios paranasais ipsilaterais), ou extensão de lesão intra-sinusal (p. ex.: acentuada hipertrofia mucosa no seio maxilar, que se estendia ao desfiladeiro óstio-infundibular). Observamos índice médio de 53,5\% de obstruções do complexo óstio-meatal (total ou parcial), correlacionado com as sinusopatias inflamatórias (Figura 11). As alterações mucosas das cavidades nasais foram observadas em 46,4\% dos casos, com maior prevalência nas crianças entre três e quatro anos de idade (Figura 12).

\section{DISCUSSÃO}

Ao iniciar-se a discussão deste trabalho, vale destacar que a casuística de 71 crianças constitui número significativo de casos, ao se comparar com a literatura consultada e a faixa etária avaliada. Em praticamente nenhum trabalho, exceto os de revisão, o número de casos foi superior ao deste trabalho, e a faixa etária analisada sempre foi maior que a nossa. 


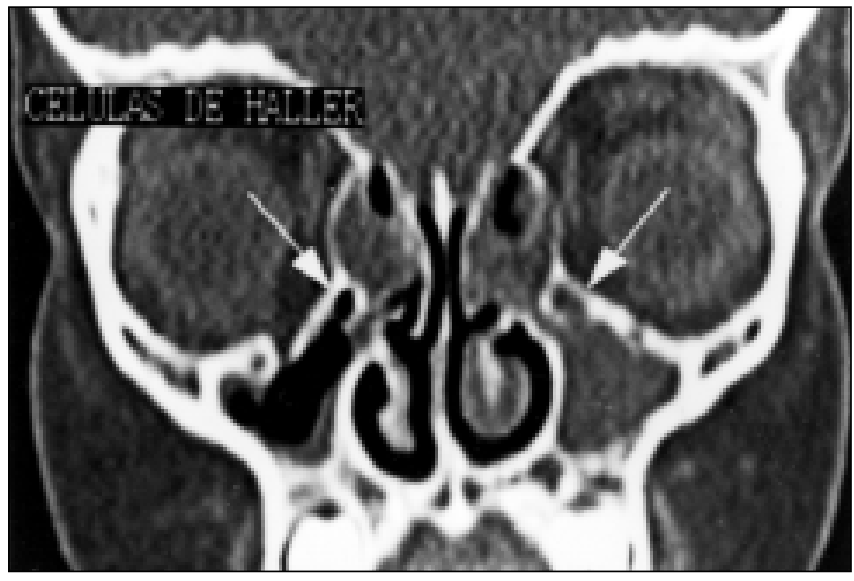

Figura 11. Plano coronal. Obstrução dos complexos óstio-meatais por hipertrofia mucosa. Existem células de Haller bilaterais, que reduzem a amplitude dos infundíbulos. Velamento total do seio maxilar direito e das células do complexo etmoidal. As alterações obstrutivas nos complexos óstio-meatais predispõem à sinusopatia inflamatória maxilo-etmoidal.

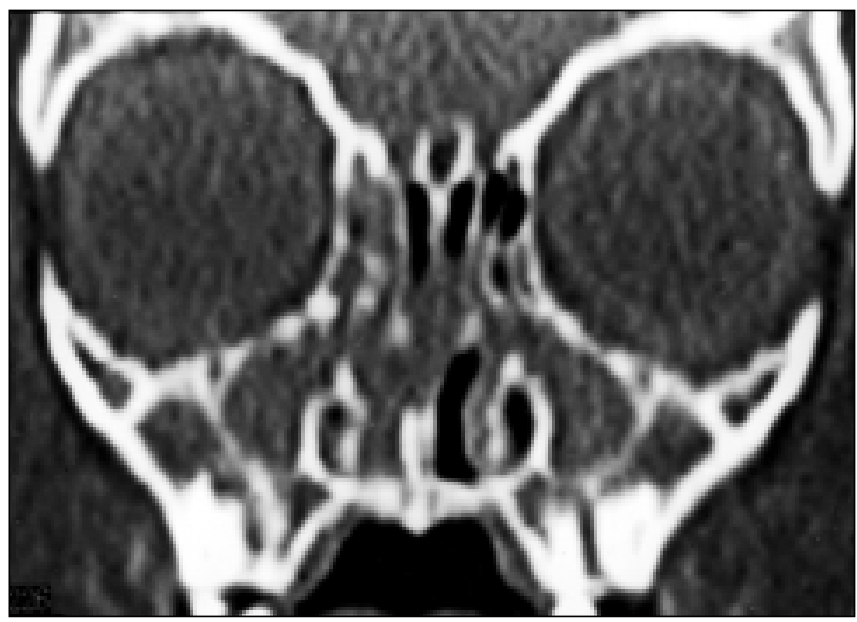

Figura 12. Plano coronal. Velamento dos antros maxilares e de células etmoidais bilateralmente e opacificação da cavidade nasal direita. "Complexo rinite-sinusite". Observa-se também obstrução dos complexos óstio-meatais, determinada por hipertrofia mucosa loco-regional.
A sinusopatia inflamatória é uma doença de grande prevalência, afetando uma entre oito pessoas nos Estados Unidos, sendo atualmente a doença crônica mais comum nesse país ${ }^{(8)}$.

As infecções das vias aéreas superiores e as alergias estão entre os principais fatores predisponentes da doença sinusal, pois iniciam alterações na superfície mucosa e na atividade ciliar dos seios paranasais, as quais predispõem à sinusite ${ }^{(5)}$. Em nosso trabalho observamos que a grande maioria das crianças era classificada como "alérgica" pelos pais, embora não existissem dados laboratoriais que comprovassem tal afirmação.

A cidade onde foi realizado este trabalho - Fortaleza, CE - é localizada na região semi-árida do Brasil, que apresenta temperatura média anual em torno de $24^{\circ} \mathrm{C}$ e não tem estações climáticas bem definidas, praticamente não existindo a estação de inverno. Desse modo, o clima pode não influenciar diretamente no desenvolvimento das sinusopatias inflamatórias, levandose em conta a amostra que foi analisada neste trabalho, ou seja, crianças com SIAR e SIC em Fortaleza, embora haja maior prevalência das infecções das vias aéreas superiores e dos processos alérgicos durante a estação de inverno e em locais de clima temperado ${ }^{(5)}$.

A maioria das crianças tem de seis a oito episódios de infecções das vias aéreas su- periores por ano ${ }^{(9)} \mathrm{e}$ a sinusite aguda é complicação em $5 \%$ a $10 \%$ dessas infecções ${ }^{(10)}$. Por conseguinte, a sinusite é mais comum em crianças do que nos adultos ${ }^{(5)}$.

Neste trabalho, a faixa etária mais prevalente foi entre três e quatro anos e houve predomínio do sexo masculino, estando de acordo com trabalhos da literatura pesquisada, como o de Lusk et al. ${ }^{(11)}$, que analisaram cerca de 115 pacientes com idade entre um e 16 anos, encontrando também predomínio do sexo masculino, e o de Casiano $^{(7)}$, que avaliou 209 pacientes com sinusopatia inflamatória crônica, com porcentuais de $53,8 \%$ para o sexo masculino e $46,2 \%$ para o sexo feminino.

A classificação em SIAR e SIC baseiase no tempo de duração dos sinais e sintomas e na recorrência deles ${ }^{(9)}$. Nas SIAR ou nas SIC o quadro clínico predominante é de descarga nasal purulenta, tosse e obstrução nasal ${ }^{(5,12,13)}$. Houve concordância entre os nossos pacientes e a literatura consultada, como o estudo de Gungor e Corey ${ }^{(5)}$, com porcentuais de $64 \%$ para rinorréia e obstrução nasal e $22 \%$ para tosse. No trabalho de Weckx et al. ${ }^{(13)}$, que analisaram crianças com idade entre três e 12 anos, os sinais e sintomas predominantes também foram a descarga e obstrução nasais (58\%) e a tosse $(22,6 \%)$, com porcentuais semelhantes aos deste trabalho, ou seja, obstrução e a descarga nasais (54 pacientes; 76\%) e a tosse (18 pacientes; 25,3\%). Em rela- ção à tosse, esta era quase sempre crônica, piorando à noite e com acessos pela manhã, logo após o despertar da criança. A secreção nasal era purulenta em cerca de $65 \%$ dos casos e a obstrução nasal era queixa muito importante entre os pacientes.

Em nosso trabalho também chamou a atenção a grande prevalência da febre $(25,3 \%)$, a qual foi um dos principais achados clínicos relatados pelos pais, embora em outros trabalhos não fosse classificada entre os mais importantes ${ }^{(5,12)}$. A febre não mais existia no momento do exame, uma vez que estas crianças já estavam sob antibioticoterapia e utilizando medicamentos anti-alérgicos e antitérmicos. A temperatura apresesentada pelas crianças durante os episódios de sinusite foi, de modo geral, em torno dos $38^{\circ} \mathrm{C}$.

Segundo outros trabalhos publicados ${ }^{\mathbf{5}}$, ${ }^{8,12)}$, a sinusite raramente é um processo isolado, e a associação de sinusopatia inflamatória e otite média foi também observada neste estudo, bem como a coexistência de hipertrofia e infecção das vegetações adenóides e das amígdalas palatinas.

A fisiopatologia da doença inflamatória sinusal é muito similar àquela das otites médias ${ }^{(12)}$. Neste trabalho, o comprometimento das orelhas médias, seja como otite secretora ou como otite média crônica, foi encontrado em 13 crianças $(18,3 \%)$, porcentual abaixo do relatado por Gungor e Corey $^{(5)}$, que descreveram entre $29 \%$ e $50 \%$ 
a correlação entre sinusopatias crônicas e otites médias de repetição.

As hipertrofias adenotonsilares causam obstruções parciais do fluxo das secreções nasossinusais, levando a um "refluxo" destas secreções para o interior dos seios paranasais, alterando o microambiente e favorecendo o crescimento bacteriano ${ }^{(5,14,15)}$.

A hipertrofia das vegetações adenóides foi muito prevalente neste trabalho, observada em $67,6 \%$ das crianças com sinusite (48 casos), com distribuição em todas as faixas etárias analisadas. A hipertrofia das amígdalas palatinas foi observada em três pacientes $(4,2 \%)$ e em todos os casos era associada à hipertrofia das adenóides.

A avaliação por métodos de imagem dos seios paranasais sempre foi baseada na radiologia convencional em suas incidências básicas (Waters, Caldwell, Hirtz e perfil). Porém, o seu valor é questionado na faixa etária infantil por causa da sua pequena correlação com as doenças do etmóide e a sub ou supervalorização dos processos inflamatórios envolvendo os seios paranasais ${ }^{(5)}$. Nas crianças com menos de dois anos de idade existem outras particularidades, como as dimensões reduzidas dos seios maxilares em relação à superfície mucosa (mucosa redundante), que tornam os achados da radiologia convencional mais duvidosos ${ }^{(\mathbf{1 6})}$.

A TC é, atualmente, o método de escolha na avaliação das doenças inflamatórias dos seios paranasais ${ }^{(\mathbf{5 , 1 7})}$, dada sua grande capacidade de distinção das diferentes densidades existentes nos seios paranasais (ar, tecidos moles e osso). Com o advento da TC helicoidal, em virtude da sua velocidade, rapidez de execução e capacidade de reconstruções multiplanares de ótima qualidade, este método tornou-se ainda mais eficaz na avaliação dos seios paranasais ${ }^{(\mathbf{1 8})}$.

Em nosso trabalho, atenção especial foi dada em relação aos parâmetros técnicos utilizados, considerando-se a exposição à radiação ionizante de um grupo de crianças de baixa idade, bem como a região do corpo avaliada. Utilizamos como parâmetros espessura de corte de $2 \mathrm{~mm}$, "pitch" 1 (velocidade de deslocamento da mesa $=2$ $\mathrm{mm} / \mathrm{s}$ ), $50 \mathrm{mAs}$ e $120 \mathrm{kV}$. Estes valores são muito menores do que aqueles apresentados na literatura mundial, como no trabalho de Suojanen e Regan ${ }^{(\mathbf{1 8})}$, que utilizaram
$2 \mathrm{~mm}$ como espessura de corte, "pitch" 1,5 (velocidade de deslocamento da mesa $=3$ $\mathrm{mm} / \mathrm{s}$ ), $165 \mathrm{mAs}$ e $120 \mathrm{kV}$. Outros artigos analisados também utilizavam técnicas de exame com maiores mAs e $\mathrm{kV}$, embora o modo de aquisição dos aparelhos não fosse espiral ${ }^{(19-21)}$. O cálculo de dose absorvida pode ser feito de maneira semelhante nos aparelhos convencionais de TC e nos de modo de aquisição espiral ${ }^{(\mathbf{2 0})}$, pois as variáveis que determinarão a dose de radiação à qual o paciente foi exposto serão o número de cortes utilizados e o tempo de exposição aos raios X. A dose de irradiação desprendida durante os exames deste trabalho pode ser considerada pequena, muito aquém daquela capaz de provocar qualquer fenômeno biológico ${ }^{(22,23)}$.

A necessidade de sedação de algumas crianças foi eventualmente questionada pelo médico assistente ou pelos pais, embora os exames fossem realizados em hospital infantil (fácil acesso à unidade de terapia intensiva, se necessário) e com equipamentos de suporte na própria sala de exame. Além disso, o medicamento utilizado (hidrato de cloral) era de fácil manuseio. Não houve qualquer incidente durante as sedações para a realização dos exames.

A aeração dos seios paranasais parece ser influenciada pelos processos inflamatórios locais, os quais atrasariam o aparecimento ou mesmo prejudicariam o desenvolvimento total destes seios. Em nosso trabalho, por exemplo, os seios do frontal só se apresentaram pneumatizados a partir dos cinco anos de idade, embora, de modo geral, a aeração destes seios se inicie em torno dos três anos de idade ${ }^{(\mathbf{2 4})}$. Nesta casuística foi demonstrado, também, que todos os seios maxilares e etmoidais encontravam-se aerados, pois estes seios iniciam o desenvolvimento já na vida fetal e talvez não sofram tão precocemente os efeitos das alterações inflamatórias.

As variações anatômicas também predispõem ou perpetuam as sinusopatias inflamatórias, sobretudo aquelas que se localizam próximas às regiões dos complexos óstio-meatais ou do recesso frontal, pois podem comprometer a ventilação e a drenagem normais dos seios, levando à doença inflamatória crônica ${ }^{(\mathbf{2 5 , 2 6})}$. São muitas e também freqüentes as variações anatômicas das cavidades nasossinusais - 52 tipos descritos - , chegando a ser encontrada alguma variação anatômica em até 93\% de pacientes submetidos a $\mathrm{TC}^{\mathbf{( 5 , 2 4 , 2 6 )}}$.

A grande maioria das variações anatômicas é assintomática e constitui "curiosidades" anatômicas, porém, o fator crítico das variações anatômicas não é a sua presença, mas o seu tamanho e suas repercussões nas regiões dos complexos óstio-meatais, reduzindo a amplitude dos óstios e canais de drenagem sinusais que compõem estas regiões $^{(\mathbf{2 6})}$.

Nesta casuística, as variações anatômicas foram demonstradas em crianças a partir de um ano de idade, embora os porcentuais totais estivessem abaixo dos encontrados por outros autores ${ }^{(\mathbf{5 , 2 6 , 2 7 )}}$. Esta menor prevalência talvez se explique pela faixa etária mais baixa avaliada neste trabalho. Houve também ótima correlação entre sinusopatia inflamatória e variações anatômicas, observando-se que em $71 \%$ dos casos que apresentavam variações anatômicas também existiam alterações sinusais compatíveis com processo inflamatório, embora Lusk et al. ${ }^{\mathbf{( 1 1 )}}$ e Laine e Smoker ${ }^{(25)}$ afirmem que estudos mais detalhados sejam necessários para se estabelecer claramente esta correlação.

As alterações encontradas na TC devem ser correlacionadas com o quadro clínico apresentado pelo paciente, como foi descrito anteriormente, pois algumas alterações da mucosa dos seios paranasais podem permanecer até alguns meses após um episódio agudo de sinusite ${ }^{(5)}$.

Os achados tomográficos observados neste trabalho mostram que o velamento total de um ou mais seios paranasais (66 casos; $92,9 \%$ ) foi muito freqüente e que os seios maxilares e etmoidais foram os mais acometidos. Outro achado freqüente foi a hipertrofia da mucosa de revestimento dos seios paranasais (48 casos; 67,6\%), que na maioria das vezes apresentava relevo irregular e era de moderada intensidade. Estes porcentuais estão em sintonia com os trabalhos analisados ${ }^{(\mathbf{5 , 2 4})}$. Gungor e Corey ${ }^{\mathbf{( 5 )}}$ relatam que $58 \%$ dos pacientes entre três e quatro anos de idade, com quadro de SIAR ou de SIC, apresentavam opacificação de seios paranasais e/ou hipertrofia da mucosa de revestimento.

Os achados tomográficos mais prevalentes (velamento sinusal e/ou hipertrofia 
mucosa) mostraram ótima correlação com o quadro clínico apresentado pelas crianças doentes, ou seja, a presença de um seio velado ou a hipertrofia da mucosa de revestimento representava realmente doença e não um achado fortuito, sem significado clínico. Alguns autores ${ }^{(2,5,28-30)}$ ainda questionam se os achados de alterações mucosas dos seios paranasais seriam indicativos de doença destes seios ou um simples achado casual. Nossa casuística reforça a idéia de que nos casos de SIAR ou de SIC as alterações mucosas são representativas de doença.

Os porcentuais de obstrução dos complexos óstio-meatais observados neste trabalho demonstram a íntima participação das disfunções desses complexos, seja do revestimento mucoso (p. ex.: reações alérgicas) ou das estruturas ósseas (p. ex.: aeração do corneto médio), na gênese das SIAR ou das SIC, como descrito por outros autores ${ }^{(\mathbf{5 , 6 , 8 )}}$. A correlação entre sinusopatia inflamatória e obstrução das regiões dos complexos óstio-meatais foi observada em $38(53,5 \%)$ dos nossos pacientes. Este porcentual talvez não tenha sido maior porque os pacientes já vinham em tratamento com antibióticos e antiinflamatórios, sendo descrito que as regiões dos complexos óstiomeatais respondem mais rapidamente que os seios paranasais ao tratamento clínico ${ }^{(\mathbf{3 0}}$ e, portanto, já estariam curadas das alterações infecciosas e inflamatórias quando da realização do exame.

$\mathrm{O}$ envolvimento das cavidades nasais ("complexo rinite-sinusite") foi muito freqüente, com porcentual de concomitância das alterações tomográficas de 46,6\% (33 pacientes). Este porcentual, assim como nos casos de obstruções dos complexos óstio-meatais associadas às sinusites, talvez fosse maior se o tratamento clínico já não estivesse instalado. A similaridade entre os revestimentos mucosos e a grande proximidade anatômica fazem com que o quadro clínico e as alterações tomográficas muito freqüentemente se sobreponham ou, às vezes, dificultem a diferenciação entre estas duas doenças, ou seja, rinite e sinusite $^{(\mathbf{1 2})}$. O envolvimento das cavidades nasais nem sempre se acompanhou de obstruções dos complexos óstio-meatais no momento da realização dos exames tomográficos, talvez pela maior rapidez de cura dos pro- cessos inflamatórios das regiões óstio-infundibulares, como já referido.

As alterações das mucosas de revestimento observadas nos exames de controle realizados em cinco pacientes, consideradas como "residuais", envolviam os seios maxilares e, sobretudo, as células etmoidais anteriores. Estas alterações "residuais" podem subseqüentemente infectar a área de drenagem do meato médio ou mesmo toda a região do complexo óstio-meatal e levar aos quadros de sinusite recorrente ou crônica, o que está de acordo com o trabalho de McAlister et al. ${ }^{\mathbf{( 2 0 )}}$.

Neste estudo, além da preocupação com o desenvolvimento de um exame rápido, calmo, com baixa dose de radiação e ótimas reconstruções multiplanares, tentou-se demonstrar que esta técnica seria útil no planejamento de cirurgia funcional endoscópica sinusal nas faixas etárias analisadas.

Trinta anos atrás, os procedimentos cirúrgicos mais freqüentes para o tratamento das SIAR e SIC eram as antrostomias e a técnica cirúrgica de Caldwell-Luc, as quais não atingiam a doença presente no complexo óstio-meatal ${ }^{(13,31)}$, o que explica os baixos índices de sucesso desses procedimentos $^{(\mathbf{2 9})}$. No final dos anos 70, Messerkling, citado por Lazar et al. ${ }^{(\mathbf{8})}$, desenvolveu o conceito de complexo óstio-meatal e alterou radicalmente o entendimento e a conduta da sinusite crônica.

A partir de sua introdução nos Estados Unidos, em 1985, por Kennedy e colaboradores, a cirurgia endoscópica sinusal funcional foi amplamente aceita e utilizada para o tratamento das sinusopatias inflama-

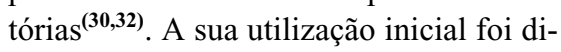
rigida à população adulta, porém em 1989 já existiam relatos e discussões sobre a sua utilização na pediatria ${ }^{(8,15,33,34)}$. Como é enfatizado na literatura ${ }^{(10,15,24,27,29,30)}$, esta cirurgia tem como objetivo a restauração das funções fisiológicas normais (drenagem mucociliar e aeração) dos seios paranasais e cavidades nasais, e a abordagem cirúrgica deve ser funcional, não com o sentido de ablação de estruturas locais, mas minimizando a ressecção de paredes ósseas e superfícies mucosas dos seios paranasais.

As indicações da cirurgia endoscópica sinusal funcional seriam as sinusopatias crônicas ou recorrentes, nas quais o tratamento clínico de longa duração não foi eficaz para erradicar a doença, polipose nasossinusal, síndrome sinobrônquica, sinusite fúngica, mucocele e piocele ${ }^{(15,17,29,35)}$.

A TC é de importância fundamental no planejamento da cirurgia endoscópica nasossinusal ${ }^{(11,15,27,36)}$. O plano coronal reproduz a visão do endoscopista e as imagens servem como "guia" para esta intervenção cirúrgica. Além disso, a TC demonstra as possíveis variações anatômicas nasossinusais, como os desvios do septo nasal, cornetos aerados ou "paradoxais", células de Haller, declives da fóvea etmoidal, alterações das lâminas papiráceas e dimensões dos seios maxilares e esfenoidais, que podem comprometer o ato cirúrgico ${ }^{(37)}$.

A técnica de exame demonstrada neste trabalho é capaz de avaliar a existência e extensão das alterações da mucosa de revestimento, o preenchimento dos seios paranasais por secreções e "débris" inflamatórios, as variações anatômicas, o estado das regiões dos complexos óstio-meatais e as estruturas circunvizinhas, o que é fundamental para o planejamento das cirurgias endoscópicas endonasais.

A utilização da cirurgia endoscópica sinusal funcional na criança ainda não é aceita por toda a comunidade médica ${ }^{(38)}$. Questiona-se se as sinusopatias teriam curso limitado, com melhora do quadro clínico ao redor dos sete anos de idade, e se os agentes infecciosos envolvidos não estariam sendo tratados inadequadamente, o que tornaria a doença recorrente ou crônica ${ }^{(38)}$.

Nos casos recidivantes e crônicos, em que tratamentos clínicos exaustivos foram tentados, e que a TC revelou alterações inflamatórias "residuais" (sobretudo etmoidais) ou extensas lesões mucosas, o tratamento cirúrgico deve ser considerado e a discussão da viabilidade do procedimento cirúrgico deve envolver o médico assistente, os pais dos pacientes e o cirurgião. Além disso, deve-se avaliar o custo social e econômico destas crianças "cronicamente" doentes e o uso contínuo de antibióticos com suas possíveis conseqüências (nefrotoxicidade, ototoxicidade, etc.).

\section{REFERÊNCIAS}

1. Marsot-Dupuch K. Indications, techniques et analyse de l'imagerie des sinus de la face. Sem Hôp Paris 1993;39:1405-15.

2. Manning SC, Biavati MJ, Phillips DL. Correlation of clinical sinusitis signs and symptoms to 
imaging findings in pediatric patients. Int J Otorhinolaryngol 1996;37:65-74.

3. Vade A, Demos TC, Olson MC, et al. Evaluation of image quality using 1:1 pitch and 1.5:1 pitch helical CT in children: a comparative study. Pediatr Radiol 1996;26:891-3.

4. Sih T. Pediatric otorhinolaryngology manual. 1st ed. New York: Saunders, 1996.

5. Gungor A, Corey JP. Pediatric sinusitis: a literature review with emphasis on the role of allergy. Otolaryngol Head Neck Surg 1997;116:4-15.

6. Yousem DM. Imaging of sinonasal inflammatory disease. Radiology 1993;188:303-14.

7. Casiano RR. Correlation of clinical examination with computer tomography in paranasal sinus disease. Am J Rhinol 1997;11:193-6.

8. Lazar RH, Younis RT, Long TE. Functional endonasal sinus surgery in adults and children. Laryngoscope 1993;103(1 Pt 1):1-5.

9. Weinberg EA, Brodsky L, Brody A, Pizzuto M, Stiner H. Clinical classification as a guide to treatment of sinusitis in children. Laryngoscope 1997; 107:241-6.

10. Isaacson G. Sinusitis in childhood. Pediatr Clin North Am 1996;43:1297-318.

11. Lusk RP, McAlister B, el Fouley A. Anatomic variation in pediatric chronic sinusitis: a $\mathrm{CT}$ study. Otolaryngol Clin North Am 1996;29:7591.

12. Parsons DS, Wald ER. Otitis media and sinusitis: similar diseases. Otolaryngol Clin North Am 1996;29:11-25.

13. Weckx LLM, Naspitz CK, Solé D, et al. Estudo clínico-laboratorial de crianças portadoras de rinite alérgica e alterações radiológicas dos seios paranasais. Rev Bras Alerg Imunol 1985;8:9-29.

14. Dunham ME. Evaluating the limited sinus computed tomography scan in children. Laryngoscope 1997;107:402-4.
15. Gross CW, Gurucharri MJ, Lazar RH, Long TE. Functional endonasal sinus surgery (FESS) in the pediatric age group. Laryngoscope 1989;99:2725

16. Weber A, May A, von Ilberg C, Halbsguth A. The value of high-resolution CT-scan for diagnosis of infectious paranasal sinuses disease and endonasal surgery. Rhinology 1992;30:113-20.

17. Rice DH. Indications for endoscopic sinus surgery. Ear Nose Throat J 1994;73:461-4, 466.

18. Suojanen JN, Regan F. Spiral CT scanning of the paranasal sinuses. AJNR 1995;16:787-9.

19. Gross GW, McGeady SJ, Kerut T, Ehrlich SM. Limited-slice CT in the evaluation of paranasal sinus disease in children. AJR 1991;156:367-9.

20. McAlister WH, Lusk R, Muntz HR. Comparison of plain radiographs and coronal CT scans in infants and children with recurrent sinusitis. AJR 1989;153:1259-64.

21. Witte RJ, Heurter JV, Orton DF, Hahn FJ. Limited axial CT of the paranasal sinuses in screening for sinusitis. AJR 1996;167:1313-5.

22. Fearon T, Vucich J. Normalized pediatric organabsorbed doses from CT examinations. AJR 1987;148:171-4.

23. Moulin G, Chagnaud C, Waultier S, et al. Radiation dose to the lenses in CT of the paranasal sinuses. Neuroradiology 1996;38 Suppl 1:S127-9.

24. Marsot-Dupuch K. Sinus de la face: inflamation. Ann Radiol (Paris) 1991;34:28-45.

25. Laine FJ, Smoker WRK. The ostiomeatal unit and endoscopic surgery: anatomy, variations, and imaging findings in inflammatory diseases. AJR 1992;159:849-57.

26. Phillips CD, Platts-Mills TAE. Chronic sinusitis: relationship between CT findings and clinical history of asthma, allergy, eosinophilia, and infection. AJR 1995;164:185-7.

27. Lusk RP, Muntz HR. Endoscopic sinus surgery in children with chronic sinusitis: a pilot study. Laryngoscope 1990;100:654-8.

28. Martin CJ, Farquhar B, Stockdale E, MacDonald S. A study of the relationship between patient dose and size in paediatric radiology. Br J Radiol 1994;67:864-71.

29. Parsons DS, Phillips SE. Functional endoscopic surgery in children: a retrospective analysis of results. Laryngoscope 1993;103:899-903.

30. Peyronny C, Bely N, Hurtier O, Bonfils P, Halimi P. Comment interpréter une TDM dans la pathologie inflammatoire des sinus. Feuillets de Radiologie 1995;5:321-34

31. Muntz HR, Lusk RP. Nasal antral windows in children: a retrospective study. Laryngoscope 1990;100:643-6.

32. Senior BA, Kennedy DW, Tanabodee J, Kroger H, Hassab M, Lanza D. Long term results of functional endoscopic sinus surgery. Laryngoscope 1998;108:151-7.

33. Mendelsohn MG, Gross CW. Soft-tissue shaver in pediatric sinus surgery. Otolaryngol Clin North Am 1997;30:443-9.

34. Shubich I, Erben D. Sinusitis crónica y cirugía funcional endoscópica-microscópica en pediatría. Reporte de 40 casos. An ORL Mex 1995;40:131-

35. Ramadan HH. Surgical causes of failure in endoscopic sinus surgery. Laryngoscope 1999;109: 27-9.

36. Oliverio PJ, Benson ML, Zinreich SJ. Update on imaging for functional endoscopic sinus surgery. Otolaryngol Clin North Am 1995;28:585-608.

37. Meyers RM,Valvassori G. Interpretation of anatomic varations of computed tomography scans of the sinuses: a surgeon's perspective. Laryngoscope 1998;108:422-5.

38. Poole MD. Pediatric sinusitis is not a surgical disease. Ear Nose Throat J 1992;71:622-3. 